\title{
Le lambeau interosseux postérieur à pédicule externe
}

\author{
Am Belmahi MD FACS, N Gharib MD, A Abbassi MD
}

\begin{abstract}
A Belmahi, N Gharib, A Abbassi. Le lambeau interosseux postérieur à pédicule externe. Can J Plast Surg 2002;10(4): 159-162.
\end{abstract}

RÉSUMÉ : Le lambeau interosseux postérieur à pédicule externe se caractérise par l'inclusion au pédicule interosseux postérieur de la quasi-totalité du fascia postérieur de l'avant-bras le lambeau ateignant la perte de substance (PDS) sans que ce pédicule ne soit tunnellisé en sous-cutané. Ses avantages par rapport à la technique classique sont :

- la couverture de PDS plus distales et plus larges au niveau de la main sans avoir ni à disséquer dangereusement la partie distale du pédicule interosseux postérieur $(\mathrm{IOP})$ ni à prélever plus de peau sur la zone donneuse par simple greffe de peau sur ce pédicule;

- le dégraissage d'emblée sans risque chez les patients obèses vu la double vascularisation septale et fasciale.

De février 1997 à mars 2001, 12 cas de lambeaux IOP à pédicule externe ont été réalisés chez des patients âgés de 16 à 65 ans. Les couvertures cutanées ont intéressé dans 8 cas la première commissure, dans 2 cas la première et la deuxième commissures et dans 2 cas les métacarpophalangiennes des 4 doigts. La taille des PDS variait de $5 \times 7 \mathrm{~cm}$ à $6 \times 12 \mathrm{~cm}$; dans tous les cas, la dissection du pédicule IOP s'est arrêtée à $2-3 \mathrm{~cm}$ de son anastomose avec le pédicule interosseux antérieur ; dans 3 cas, un dégraissage d'emblée du lambeau a été réalisé sans souffrance vasculaire ultérieure. Tous ces patients ont bénéficié en postopératoire d'une attelle main- tenant le poignet en extension pendant 3 semaines date à laquelle le pédicule a été sectionné. Aucune complication n'est à déplorer.

Mots clés : Dégraissage d'emblée, Fascia postérieur de l'avant bras; Lambeau interosseux postérieur

\section{The posterior interosseous flap with external pedicle}

The posterior interosseous flap with external pedicle is characterized by the inclusion of the quasi-totality of the posterior forearm fascia to the septum of the posterior interosseous vessels, the flap reaching the defect without tunnelization of this pedicle. The coverage of distal defects in the hand without dangerous dissection of the distal part of the interosseous posterior pedicle, the coverage of large skin defects without sacrificing more skin in the donor area by simply grafting this pedicle, and the possibility of secure immediate defatting of this flap are the main advantages of this technique. From February 1997 to March 2001, there were 12 cases of PIO flaps with external pedicle in patients aged 16 to 65 years. This skin coverage involved the first web space in eight cases, the first and second web spaces in two cases, and the dorsal side of the metacarpophalangeal joints in two other cases. These skin defects were due to severe retractile sequellae of thermic and electrical burns, and their sizes varied from $5 \times 7$ $\mathrm{cm}$ to $6 \times 12 \mathrm{~cm}$. In all cases, the dissection of the posterior interosseous pedicle stopped $2 \mathrm{~cm}$ before its anastomosis with the anterior interosseous artery. In three cases of obese patients, immediate defatting was peformed without any complications. All patients benefitted from an orthesis that kept the wrist in extenstion for three weeks to protect the pedicle, which was sectionned at this date. No complications were reported.

Service de Chirurgie Plastique et Chirurgie de la main, Hôpital Avicenne, Rabat, Maroc

Demandes de renseignements et tirés à part : Pr Am Belmahi, 9 rue Ghzaoua, Rabat, Souissi, Maroc. Telephone 011-212-7-61-15-30-38, fax 011-212-7-37-67-46-18, e-mail belmahi@wanadoo.net.ma 
T e lambeau interosseux postérieur (IOP) aura bientôt 13 Lans $(1,2,3)$ et reste toujours très utilisé dans la couverture des pertes de substance cutanée de la main $(4,5)$. Cependant, tous les auteurs s'acharnent à tunnelliser le pédicule du lambeau sous un pont cutané de manière à fermer hermétiquement la PDS (6). Nous rapportons une variante technique, «le lambeau IOP à pédicule externe» qui se caractérise par l'inclusion au septum interosseux postérieur du fascia de la face postérieure de l'avant-bras et qui a 3 principaux avantages par rapport à la technique classique :

- la possibilité de couvrir des PDS distales de la main (niveau interphalangien proximal des doigts [IPP]) sans avoir à disséquer dangereusement la partie distale du pédicule IOP;

- la couverture de larges PDS de la main sans prélèvement important de peau sur la zone donneuse;

- le dégraissage d'emblée sans risque chez les patients obèses.

Nous l'avons utilisée chez 12 patients depuis février 1997 avec une satisfaction totale tant sur le plan de la couverture que sur celui des séquelles.

\section{MATÉRIEL ET MÉTHODES}

\section{Matériel}

12 patients (6 hommes et 6 femmes) âgés de 16 à 65 ans, tous porteurs de séquelles rétractiles graves, secondaires à des brûlures thermiques dans 10 cas et électriques dans 2 cas, ont bénéficié d'un lambeau IOP à pédicule externe pour couvrir les PDS cutanées en rapport avec la libération de ces rétractions qui a constamment exposé les structures nobles de la main. Ces lambeaux ont été réalisés de février 1997 à mars 2001. Les PDS cutanées avaient une taille variant de 5 à $7 \mathrm{~cm}$ de large sur 6 à $12 \mathrm{~cm}$ de long mais la taille de la palette cutanée n'a jamais excédé $5 \mathrm{~cm}$ de large sur 8 $\mathrm{cm}$ de long. Ces PDS ont intéressé dans 8 cas la première commissure, dans 2 cas la première et la deuxième commissures et dans 2 autres cas la face dorsale des métacarpophalangiennes des 4 doigts ainsi que celle de la main; dans ces 2 derniers cas, une greffe de peau mince a été appliquée sur le large pédicule du lambeau.

\section{Méthodes}

Les repères de la dissection de ce type de lambeau sont les mêmes que ceux de Zancolli et Masquelet ; la technique de levée de ce lambeau est identique à celle d'un lambeau IOP classique sauf que la quasi-totalité de l'aponévrose de la face postérieure de l'avant-bras est incluse au septum du lambeau (figure 1, gauche) ; la dissection distale du pédicule s'arrête à $2 \mathrm{~cm}$ de l'anastomose avec le pédicule interosseux antérieur sur la face dorsale du poignet (figure 1, droite). Le pédicule IOP associé au fascia n'est pas tunnellisé sous un pont cutané (figure 1, centre) mais le lambeau en îlot ferme quasi-hermétiquement la PDS (figures 2-4). Ce pédicule externe peut être greffé en peau mince pour élargir les possibilités de couverture sans plus de sacrifice cutané. L'extension du poignet en postopératoire à $50^{\circ}$ est maintenue par une atelle plâtrée et le pédicule protégé par un pansement gras pendant 3 semaines, date à laquelle le sevrage est fait sous anesthésie purement locale. Chez 3 patients obèses, le dégraissage d'emblée du lambeau a été réalisé en respectant $5 \mathrm{~mm}$ de part et d'autre du septum (figures 2-4).

\section{RÉSULTATS}

- Aucune souffrance vasculaire sur ce lambeau IOP à pédicule externe n'a été observé lors du sevrage du pédicule; de même, la prise de la greffe de peau mince dans les 2 cas où le fascia a été greffé a été totale et n'a pas été altérée par ce sevrage.

- Les 3 cas de dégraissage d'emblée du lambeau n'ont pas posé de problème vasculaire particulier.

- Avec un recul moyen de 23 mois (extrêmes: 2 mois à 48 mois), toutes les couvertures cutanées obtenues sont stables et de très bonne qualité.

- Aucun problème de raideur articulaire du poignet n'existe 2 mois après le sevrage.

- Les séquelles liées au prélèvement du fascia de la face postérieure de l'avant-bras sont inéxistants.

\section{DISCUSSION}

Le lambeau IOP à pédicule externe se définit de 2 manières :

- Sur le plan anatomique, il inclut dans la dissection le fascia de la quasi-totalité de la face postérieure de l'avant-bras au septum du pédicule IOP.

- Sur le plan hémodynamique, à sa vascularisation septale $(7,8)$ s'ajoute une vascularisation fasciograisseuse tributaire du fascia prélevé. Ce fascia a pour nous un double rôle physiologique :

- Il protège tout d'abord le pédicule IOP externe voué à une dessication certaine s'il n'est pas englobé dans une structure tissulaire bien vascularisé.

- Il améliore aussi la vascularisation de la palette cutanée puisqu'on ajoute à la vascularisation septale une vascularisation fasciale ; c'est d'ailleurs cette sécurité vasculaire qui nous a permis de réaliser avec succès, d'emblée, et à 3 reprises le dégraissage de ce lambeau chez des patients obèses; des plasties en $\mathrm{Z}$ d'intégration peuvent également être associées, pour les mêmes raisons, rendant le lambeau IOP à pédicule externe un lambeau «tout en un temps»(sevrage exclu). 

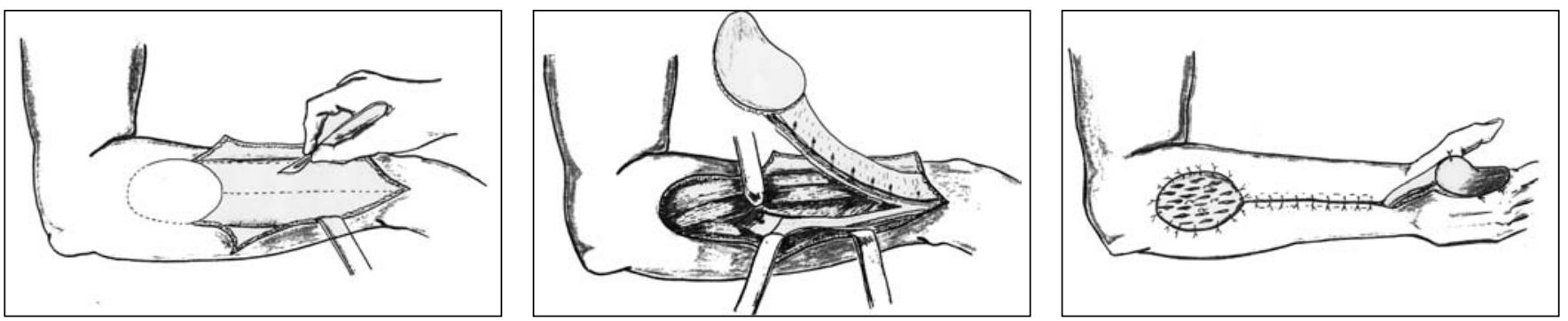

Figure 1) Gauche Les repères de levée du lambeau interosseux postérieur à pédicule externe sont ceux de Masquelet et Zancolli. Le fascia de la face postérieure de l'avant-bras est prélevé en totalité. Centre La dissection distale du pédicule interosseux postérieur s'arrête à $2 \mathrm{~cm}$ de son anastomose avec le pédicule interosseux antérieur sur la face dorsale du poignet. Droite Le septum interosseux postérieur et le fascia de la face postérieure de l'avant-bras ne sont pas tunnellisés sous un pont cutané pour atteindre la perte de substance cutané
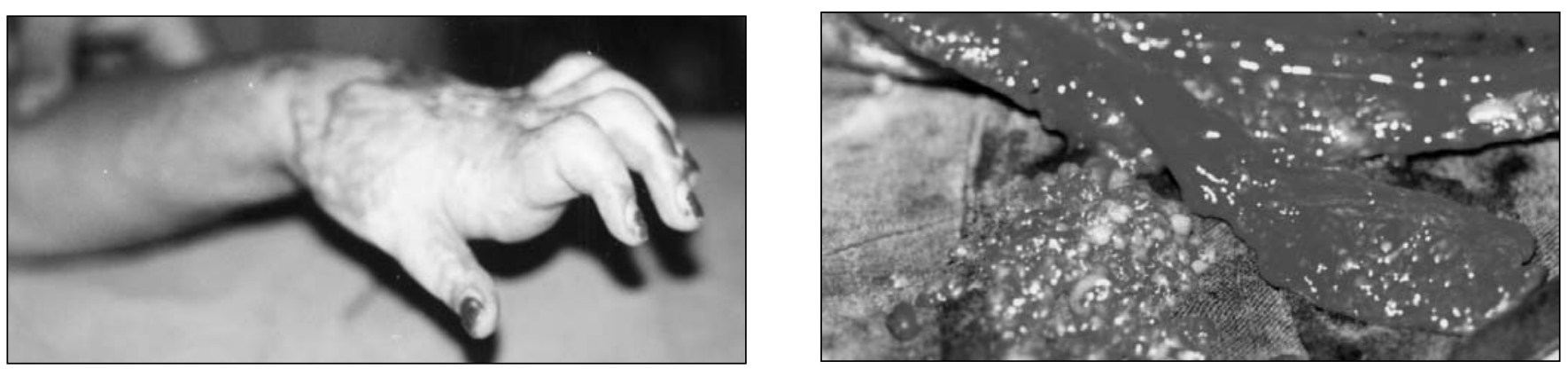

Figure 2) Gauche Séquelle rétractile grave de brûlure thermique. Rétraction sévère de la 1ère commissure -Aspect de main araignée. Droite Levée d'un lambeau I.O.P à pédicule externe pour couvrir la perte de substance cutané secondaire à la libération de la rétraction de la 1 ère commissure; il est dégraissé d'emblée. Les rétractions digitales sont traitées par des ténoarthrolyses et des greffes de peau totale en unités fonctionnelles
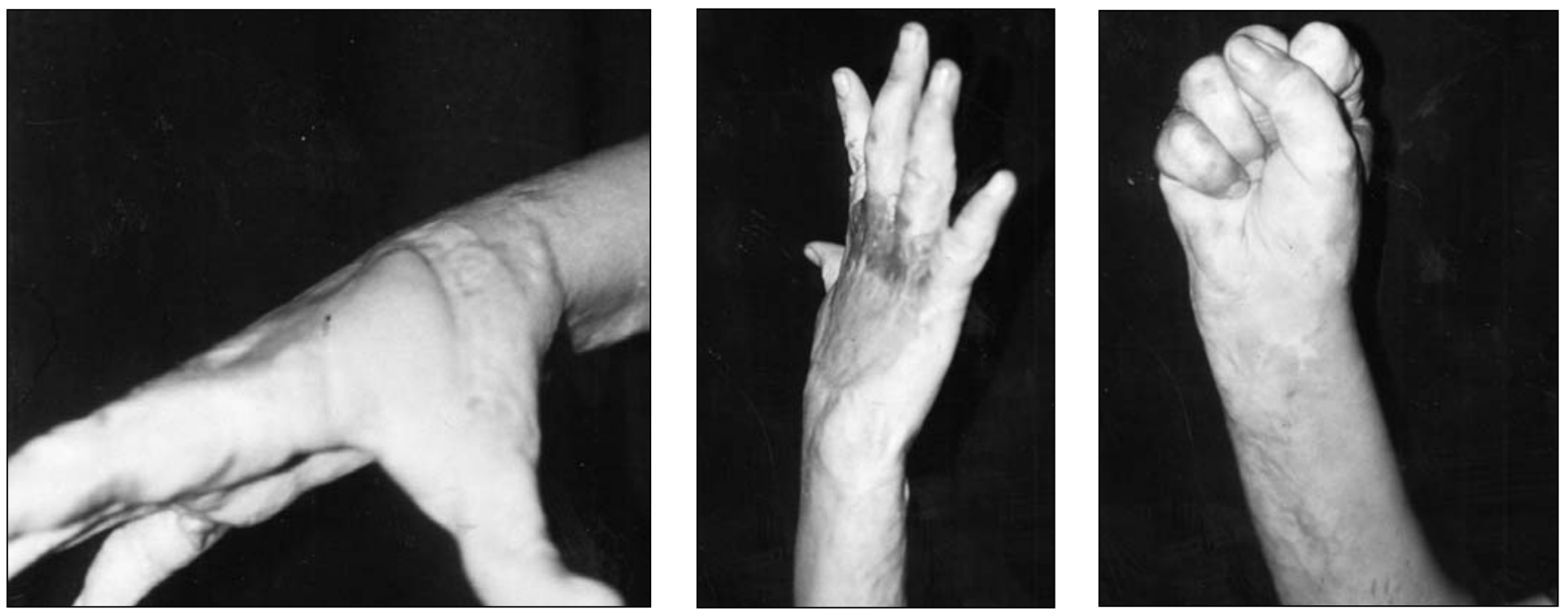

Figure 3) Gauche, Centre, Droite Résultats 3 ans aprés la correction des différentes rétractions

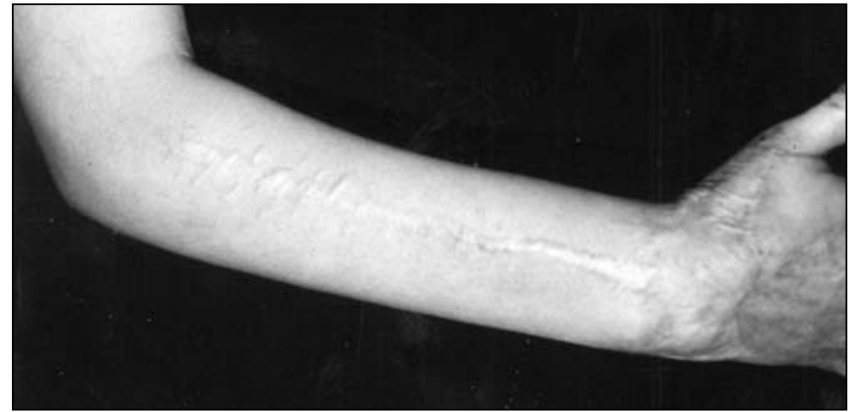

Figure 4) Séquelles de la zone donneuse du lambeau au bout de 3 ans
Sur le plan purement plastique, ce pédicule externe nous paraît très intéressant pour 3 raisons :

- Il permet au lambeau d'atteindre des PDS des doigts sans avoir à disséquer dangereusement, comme dans un lambeau IOP classique, la partie distale du pédicule IOP jusqu'à sa jonction avec le pédicule interosseux antérieur (IOA) (9,10,11). En effet, le pédicule externe joint à l'extension du poignet pendant 3 semaines permet de couvrir ces PDS en restant à $2 \mathrm{~cm}$ de cette anastomose. 
- La couverture de larges PDS au niveau de la main et des doigts peut se faire sans grand sacrifice cutané, le fascia étant greffé en peau mince ; la section du pédicule à la 3ème semaine n'altère en rien la prise de la greffe.

- Le dégraissage d'emblée est sans risque chez les patients obèses.

Ces avantages nous font préférer le lambeau IOP à pédicule externe à la technique classique dans les cas de PDS distales sur la main, larges et chez les sujets obèses.

Certains auteurs tel que Foucher (12) ont utilisé le même principe pour des lambeaux en îlot à haut flux rétrograde tel que le « lambeau chinois à pédicule externe » en emportant uniquement une atmosphère cellulo-graisseuse autour du pédicule ; le dégraissage et les plasties en $\mathrm{Z}$ d'intégration n'ont pas altéré la vascularisation de ce lambeau. D'autre ont dégraissé d'emblée des lambeaux fascio-cutanés

\section{RÉFÉRENCES}

1. Zancolli EA, Angrigiani C. Posterior interosseous island forearm flap. J Hand Surg 1988;138:130-5.

2. Zancolli A, Angrigiani C. Colgajo dorsal de antebraso (" en isla ") (pediculo de vasos interoseos posteriores). Rev Asoc Arg Ortop Traumato 1986;51:161-8.

3. Masquelet AC, Penteado CV. Le lambeau interosseux postérieur. Ann Chir Main 1987;6:131-9.

4. Costa H, Smith R, McGrouther DA. Thumb reconstruction by the posterior interosseous osteocutaneous flap. Br J Plast Surg $1988 ; 41: 228-33$.

5. Costa H, Soutar DS. The distally based island posterior interosseous flap. Br J Plast Surg 1988;41:221-7.

6. Foucher $G$, Merle $M$. Le lambeau antebrachial de làrtère radiale en chirurgie de la main. In: Gilbert A, Masquelet AC, Henta RV. Les Lambeaux Artériels Pédicules de Membre Supériur. Expansion Scientifique Francaise, 1991:72-8. à vascularisation axiale tel que le lambeau inguinal sans nécrose cutanée.

Certes, des inconvénients existent pour le lambeau IOP à pédicule externe :

- Le patient doit garder pendant 3 semaines une attelle plâtrée maintenant le poignet en extension ; lors de son retrait, la petite raideur en extension du poignet disparaît totalement à la fin du 2ème mois.

Le pédicule externe nécessite des soins journaliers par pansement gras.

Il est à signaler que le sacrifice de la totalité du fascia postérieur de l'avant-bras n'a de conséquences ni sur le plan fonctionnel ni sur le plan esthétique pour l'avant-bras.

En définitive, pour nous, le lambeau IOP classique reste très indiqué pour les petites pertes de substance proximales au niveau de la main chez les patients minces mais, dans les situations contraires, le lambeau IOP à pédicule externe a notre préférence.

7. Penteado CV, Masquelet AC, Chevrel JP. The anatomic basis of the fascio-cutaneous flap of the posterior interosseaus artery. Surg Radiol Anat 1986;8:209-15.

8. Salmon M. Artères de la peau. Paris: Masson, 1936.

9. Hu W, Martin D, Foucher G. Le lambeau interosseux antérieur. Annales de Chirurgie Plastique Esthétique 1994;39:290-300.

10. Hu W. Le lambeau interosseux antérieur en îlot à cantrario : étude anatomique et application en chirurgie de la main. Mémoire; Université Pierre et Marie Curie. Faculté de médecine Paris IV, 1991.

11. Hu W, Martin D, Baudet J. Reconstruction de la colonne du pouce par les lambeaux ostéo-cutanés de l'avant bras. Ann Chir Plast Esthet 1993;38:381-91.

12. Braun FM, Hoang Ph, Merle M, Van Genechten F, Foucher G. Techniques et indications du lambeau antebrachial en chirurgie de la main; à propos de 33 cas. Ann Chir Main 1985;4:85 . 